12

\title{
Эрозия переднего фронта низкоэнергетического пучка при инжекции в газ во внешнем магнитном поле
}

\author{
() В.П. Григорьев, И.Л. Звигинцев
}

Томский политехнический университет, 634034 Томск, Россия

e-mail: Zvigintsev@yandex.ru

(Поступило в Редакцию 26 апреля 2017 г.)

Проведены исследования эрозии переднего фронта низкоэнергетического сильноточного электронного пучка при инжекции его в нейтральный газ низкого давления во внешнем магнитном поле. Определены параметры системы, при которых образуется виртуальный катод, длительность эрозии переднего фронта пучка и время полной зарядовой нейтрализации.

DOI: 10.21883/JTF.2018.05.45905.2311

\section{Введение}

В настоящее время при решении технологических проблем, связанных с изменением состояния и свойств поверхности материалов и синтезом органических и неорганических соединений в плазме [1-3], широко используются низкоэнергетические сильноточные электронные пучки (СЭП) с энергией электронов $\varepsilon_{b} \sim 10-40 \mathrm{keV}$ и высокой плотностью тока $j_{b}>20 \mathrm{~A} / \mathrm{cm}^{2}$.

Перспективность использования таких электронных пучков обусловлена их способностью переносить запасенную энергию на достаточно большие расстояния к объекту воздействия $[4,5]$. При этом одной из наиболее важных задач является минимизация энергетических потерь пучка при его транспортировке к мишени. Так как транспортировка СЭП из технологических требований обычно осуществляется в слабо ионизованной плазме или нейтральном газе низкого давления (менее 0.1 Torr), то одной из основных причин потерь является эрозия переднего фронта пучка, обусловленная недостаточной степенью нейтрализации заряда пучка в начальные моменты транспортировки.

Исследование этой проблемы достаточно полно проведено для релятивистских пучков [6,7]. В этих работах показано, что эрозия переднего фронта пучка в основном связана с расхождением пучка и потерей заряда на стенке трубы дрейфа. Очевидно, что этот эффект может быть подавлен при наложении достаточно сильного внешнего продольного магнитного поля.

В случае низкоэнергетических СЭП имеются существенные особенности, обусловленные образованием на входе в трубу дрейфа виртуального катода (ВК). Действительно, при отсутствии зарядовой нейтрализации такой электронный пучок в результате образования ВК практически полностью запирается на входе в пространство дрейфа. При этом внешнее магнитное поле не влияет на потери тока.

В рамках настоящей работы проводится исследование и определение основных закономерностей эрозии перед- него фронта низкоэнергетических СЭП при инжекции в трубу дрейфа, заполненную нейтральным газом, во внешнем магнитном поле на основе математической модели, в которой учитывается образование ВК и ионизация электронами пучка и плазмы, проводится анализ динамики наработки плазменного канала.

\section{Основные уравнения модели}

При инжекции электронного пучка в нейтральный газ основными процессами формирования плазменного канала являются ионизация электронами пучка и ионизация образующимися электронами плазмы в поле пространственного заряда:

$$
\begin{gathered}
\frac{\partial n_{i}}{\partial t}=\sigma_{i b} v_{b} n_{p r} n_{g}+\sigma_{i e} v_{e} n_{e} n_{g}, \\
\frac{\partial n_{e}}{\partial t}=\frac{\partial n_{i}}{\partial t}-n_{e} \frac{v_{e}}{L},
\end{gathered}
$$

где $n_{i}, n_{e}$ - плотности ионов и электронов плазмы, $\sigma_{i b}, \sigma_{i e}-$ сечения ионизации электронами пучка и плазмы, $v_{b}, v_{e}$ - скорости электронов пучка и плазмы, $n_{p r}$ - плотность проходящего пучка, $n_{g}=$ $=\left(3.5 \cdot 10^{16} p-n_{i}\right)-$ плотность газа, $p$ - давление газа в торах, $L$ - длина трубы дрейфа.

В начале импульса при достаточно низком токе через трубу дрейфа пучок проходит полностью. С ростом тока пучка, когда выполняется неравенство $\left|I_{b}\right|>\left|I_{p r}^{(0)}+I_{b} f\right|$, образуется ВК, где $I_{b}$ - ток инжектируемого пучка, $I_{p r}^{(0)}$ - предельный ток в вакууме, $f=n_{i} / n_{b}-$ степень зарядовой нейтрализации $\left(f=1\right.$ при $\left.n_{i}>n_{b}\right)$, $n_{b}$ - плотность электронов инжектируемого пучка [8]. За ВК проходит ток электронов $I_{p r}$, равный $I_{p r}^{(0)}+I_{b} f$. Остальная часть тока инжектируемого пучка отражается от ВК. Так как для типичных экспериментальных параметров $[9,10]$ радиус трубы дрейфа в 2-3 раза превышает радиус пучка, тормозящее поле $E_{z}$ слабо меняется по сечению пучка. Поэтому в хорошем 
приближении можно считать плотность проходящего тока однородной, а предельный ток будет иметь вид [8] $\left|I_{p r}^{(0)}\right|=(2 / 9) I_{A}^{(0)} \beta^{3} /\left[1+(4 / 3) \ln \left(R_{c} / r_{b}\right)\right]^{3 / 2}$, где $I_{A}^{(0)}=c e / r_{0} \approx 17 \mathrm{kA}, c-$ скорость света в вакууме, $e$ - элементарный заряд, $r_{0}$ - классический радиус электрона, $\beta=v_{b} / c$ - относительная скорость пучка, $r_{b}$ и $R_{c}-$ радиусы пучка и трубы дрейфа.

Одновременно с инжекцией электронного пучка происходит ионизация газа, описываемая уравнением (1). Первое слагаемое в правой части этого уравнения отвечает за ионизацию газа электронами пучка, второе за ионизацию плазменными электронами. Уравнение (2) описывает баланс электронов в пространстве дрейфа. Первое слагаемое описывает рождение электронов, эквивалентное ионам, второе - выход плазменных электронов на правом торце трубы дрейфа под действием пространственного заряда.

При наличии достаточно сильного внешнего магнитного поля движение плазменных электронов, образующихся при ионизации газа электронами пучка, в основном происходит в поле, связанном с образованием ВК, который образуется вблизи плоскости инжекции на расстоянии порядка $d=$ $=\sqrt{(\sqrt{2} /(9 \pi))\left(I_{A}^{(0)} / j_{b}\right)\left(\varepsilon_{b} / E_{0}\right)^{3 / 2}}[11]$, где $j_{b}-$ плотность тока инжектируемого пучка, $\varepsilon_{b}$ - энергия электронов пучка, $E_{0}$ - энергия покоя электрона. Потенциал ВК $U$ соответствует энергии электронов пучка, а торцы трубы дрейфа заземлены. При этом электроны плазмы, ускоряясь полем ВК, проходят в среднем разность потенциалов $U / 2$ за время $L / v_{e}$. Поэтому будем полагать среднюю энергию электронов плазмы равной половине энергии электронов пучка $\left(\varepsilon_{e}=\varepsilon_{b} / 2\right)$. Тогда $\sigma_{i b}=\sigma_{i}\left(\varepsilon_{b}\right), \sigma_{i e}=\sigma_{i}\left(\varepsilon_{e}\right), v_{b}=v\left(\varepsilon_{b}\right)$, $v_{e}=v\left(\varepsilon_{e}\right)$, где $\sigma_{i}$ - сечение ионизации атомов газа [12], $v(\varepsilon)=c \sqrt{1-\left(1+\left(\varepsilon / E_{0}\right)\right)^{-2}}$ - зависимость скорости электронов от энергии.

\section{Результаты}

Численное исследование модели проводилось для диапазона параметров $\left|I_{b 0}\right|=0.3-15 \mathrm{kA}, \quad B_{z}=$ $=1.5 \mathrm{kG}, \varepsilon_{b}=10-90 \mathrm{keV}, \tau=160 \mu \mathrm{s}, t_{f}=20 \mu \mathrm{s}, \quad p=$ $=10^{-4}-10$ Torr, $r_{b}=2 \mathrm{~cm}, \quad R_{c}=4.1 \mathrm{~cm}, L=20 \mathrm{~cm}$, $\mathrm{Ar}$ и Не. В качестве импульса использовался модельный импульс однородного пучка, состоящий из переднего фронта, полочки и заднего фронта (рис. 1). Радиальное распределение определялось по формуле $\quad j_{b}=\left(\exp \left(-\left(r / r_{b}\right)^{16}\right)-\exp \left(-\left(R_{c} / r_{b}\right)^{16}\right)\right) j_{b 0}$, где $j_{b 0}=j_{b}(r=0)$, которое соответствует однородному пучку со сглаженной границей. Вычисления проводились до достижения полной зарядовой нейтрализации.

Типичная временна́я зависимость эрозии переднего фронта пучка показана на рис. 2. Здесь отчетливо видны

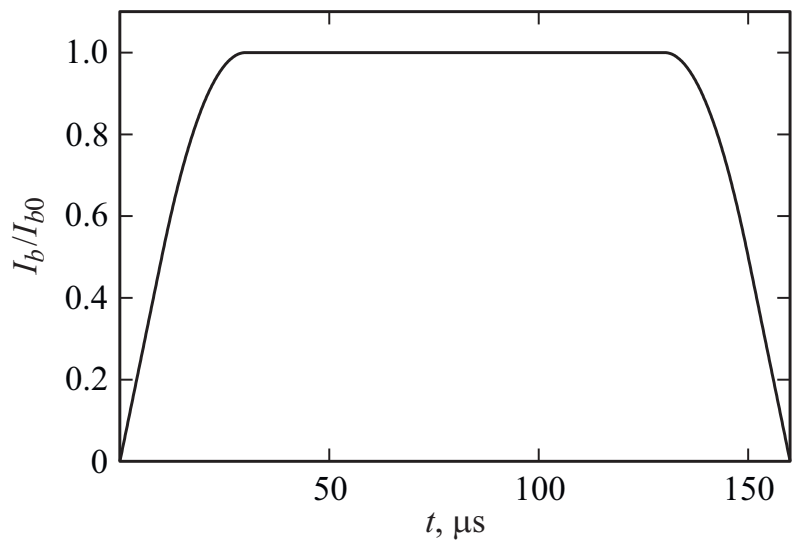

Рис. 1. Временно́й профиль инжектируемого пучка.

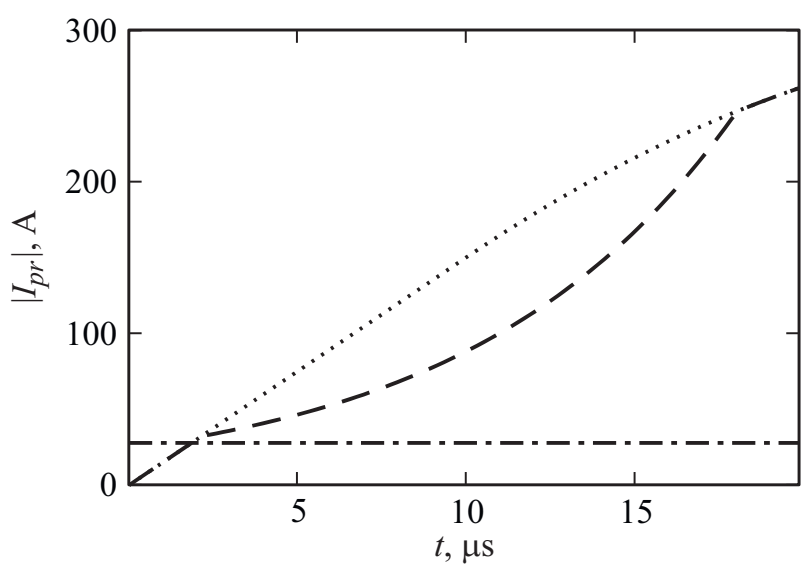

Рис. 2. Эрозия переднего фронта пучка (штриховая - $\left|I_{p r}\right|$, пунктирная - $\left|I_{b}\right|$, штрихпунктирная $\left.-I_{p r}^{(0)}=27.8 \mathrm{~A}\right)$; $p=10^{-4}$ Torr, $\quad r_{b}=2 \mathrm{~cm}, \quad R_{c}=4.1 \mathrm{~cm}, \quad I_{b 0}=-300 \mathrm{~A}$, $\varepsilon_{b}=20 \mathrm{keV}$, Ar.

три характерные области: до образования ВК, существование ВК (проходящий ток ниже тока пучка) и после исчезновения ВК. Длительность каждой из областей может быть различной. Так, при параметрах, указанных на рисунке, длительность эрозии в аргоне составляет $15 \mu \mathrm{s}$, а время полной зарядовой нейтрализации $20 \mu \mathrm{s}$. Как показывают численные расчеты, увеличение энергии до $30 \mathrm{keV}$ приведет к уменьшению времени эрозии до $10 \mu \mathrm{s}$. А при $50 \mathrm{keV}$ эрозия вообще не возникнет.

На рис. 3 показана эрозия переднего фронта пучка с энергией $70 \mathrm{keV}$ при инжекции в гелий. Видно, что эрозия в гелии существует и при относительно высоких энергиях электронов пучка. Это обусловлено менее эффективной ионизацией гелия по сравнению с аргоном. Для приведенных параметров (рис. 3) эрозия исчезает только при энергиях порядка $100 \mathrm{keV}$.

В зависимости от энергии электронов пучка предельный ток будет меняться. От его величины зависит промежуток времени до образования ВК, а также промежуток времени до полной зарядовой нейтрализации пучка. 


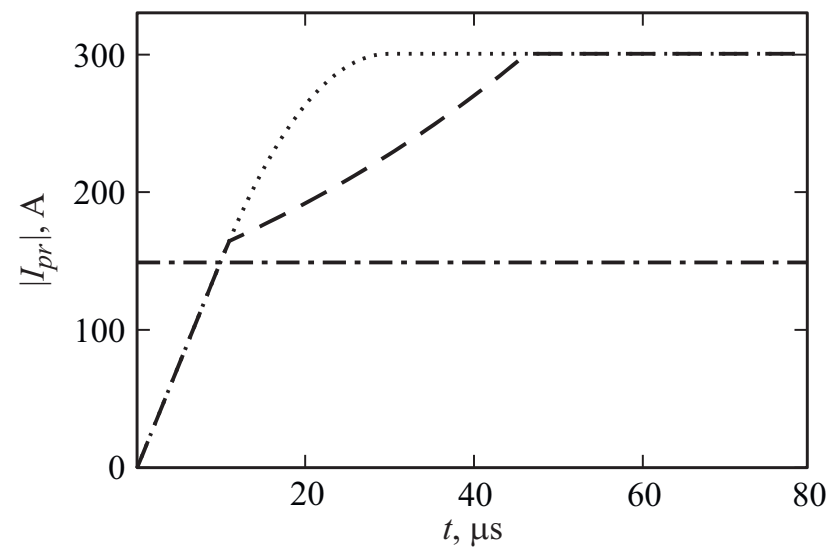

Рис. 3. Эрозия переднего фронта пучка (штриховая - $\left|I_{p r}\right|$, пунктирная $-\left|I_{b}\right|$, штрихпунктирная $\left.-I_{p r}^{(0)}\right) ; p=10^{-4}$ Torr, $r_{b}=2 \mathrm{~cm}, R_{c}=4.1 \mathrm{~cm}, I_{b 0}=-300 \mathrm{~A}, \varepsilon_{b}=70 \mathrm{keV}$, He.

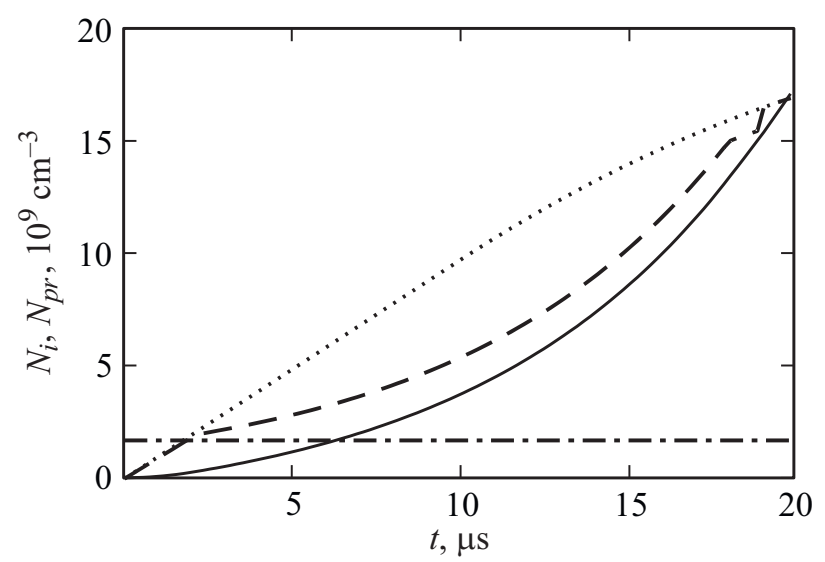

Рис. 4. Временнб́е зависимости плотностей ионов плазмы (сплошная), проходящих электронов пучка (штриховая) и инжектируемого пучка (пунктирная), штрихпунктирная соответствующая предельному току плотность электронов $\left(n_{p r}^{(0)}=1.7 \cdot 10^{9} \mathrm{~cm}^{-3}\right) ; p=10^{-4}$ Torr, $r_{b}=2 \mathrm{~cm}, R_{c}=4.1 \mathrm{~cm}$, $I_{b 0}=-300 \mathrm{~A}, \varepsilon_{b}=20 \mathrm{keV}$, Ar.

Время полной зарядовой нейтрализации $\left(\tau_{n}\right)$ как в аргоне, так и в гелии, имеет сложную зависимость от энергии электронов пучка при наличии эрозии. Это связано с зависимостью сечения ионизации и предельного тока от энергии электронов пучка. В отсутствие эрозии время полной зарядовой нейтрализации возрастает с ростом энергии электронов пучка, так как предельный ток в этом случае не оказывает никакого влияния, а сечение ионизации уменьшается. Так, с ростом энергии от 45 до $100 \mathrm{keV}$ в аргоне при $I_{b 0}=-300 \mathrm{~A}, p=10^{-4}$ Torr, $r_{b}=2 \mathrm{~cm}, R_{c}=4.1 \mathrm{~cm}$ время полной зарядовой нейтрализации возрастает от 20 до $24 \mu \mathrm{s}$.

Во время существования ВК, разница между плотностью проходящих электронов пучка и плотностью ионов равна плотности электронов, соответствующей предельному току (рис. 4). При указанных на рисунке параметрах полная зарядовая нейтрализация достигается в конце переднего фронта пучка. Увеличение давления до $10^{-3}$ Torr приведет к уменьшению $\tau_{n}$ до $1.6 \mu \mathrm{s}$, а ВК образовываться не будет.

В процессе наработки плазменного канала происходит накопление ионов в пространстве дрейфа. Электроны плазмы постоянно выходят из трубы дрейфа через правый торец за счет поля ВК. Они имеют конечную скорость выхода, за счет этого в трубе дрейфа содержится ненулевая плотность электронов плазмы. Но она достаточно мала по сравнению с плотностью ионов плазмы (рис. 5). И с течением времени разница между плотностями ионов и электронов плазмы только увеличивается. Сравнивая рис. 4 и 5 , видно, что плотность электронов плазмы на три порядка меньше плотности ионов. Уменьшение скорости плазменных электронов приведет к увеличению их плотности в пространстве дрейфа. Так, если взять среднюю энергию плазменных электронов равной $5 \mathrm{eV}$, оставляя остальные параметры без изменения, то в момент достижения полной зарядовой нейтрализации плотность ионов достигнет $1.7 \cdot 10^{10} \mathrm{~cm}^{-3}$, а плотность электронов плазмы будет равна $3.2 \cdot 10^{8} \mathrm{~cm}^{-3}$. Увеличение длины трубы дрейфа в 5 раз приведет к увеличению плотности плазменных электронов в 5 раз.

Из рис. 5 видно, что профиль плотности плазменных электронов качественно совпадает с профилем плотности проходящего за ВК пучка. Это действительно так, когда время выхода плазменных электронов из трубы дрейфа достаточно мало. В этом случае кривая 1 рис. 5 представляет собой плотность электронов, которые рождаются в результате ионизации в текущий момент, пропорциональную плотности электронов проходящего тока пучка. При меньших энергиях электронов пучка ионизация газа происходит интенсивнее за счет большего сечения ионизации, что и видно на рис. 5 .

На рис. 6 и 7 представлены обобщенные результаты численных исследований по определению границы образования ВК при изменении различных параметров. Для

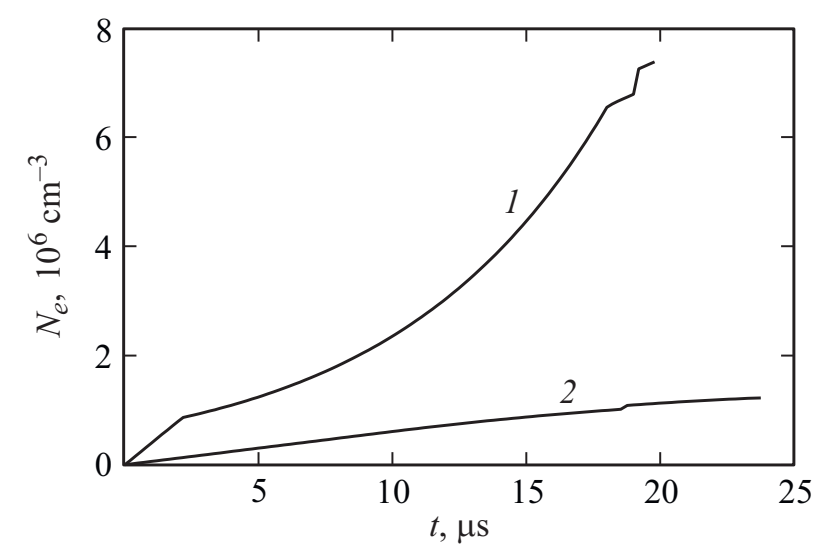

Рис. 5. Временна́я зависимость плотности электронов плазмы до момента полной зарядовой нейтрализации пучка; $p=10^{-4}$ Torr, $r_{b}=2 \mathrm{~cm}, R_{c}=4.1 \mathrm{~cm}, I_{b 0}=-300 \mathrm{~A}$, Ar. Кривая $1-\varepsilon_{b}=20$, кривая $2-\varepsilon_{b}=90 \mathrm{keV}$. 
каждой кривой образованию ВК соответствуют точки в пространстве параметров, лежащие ниже соответствующей кривой. На рис. 6 каждая кривая соответствует фиксированному значению амплитуды тока пучка. Из поведения кривых на рис. 6 видно, что с ростом амплитуды тока пучка необходимо увеличивать давление газа и энергию электронов пучка, чтобы избежать эрозии переднего фронта.

На рис. 7 каждая кривая соответствует фиксированному значению энергии электронов пучка. Из рисунка видно, что в килоамперных пучках возникновение эрозии линейно зависит от силы тока пучка. При $\varepsilon_{b}=10 \mathrm{keV}$ и $I_{b 0}=-15 \mathrm{kA}$ требуется давление газа свыше $9 \cdot 10^{-2}$ Torr, чтобы ВК не образовывался.

Возьмем границы образования ВК на рис. 6 и для каждой точки на этих границах определим время достижения полной зарядовой нейтрализации. В результате получим графики, представленные на рис. 8. Видно, что увеличение амплитуды тока пучка и уменьшение энер-

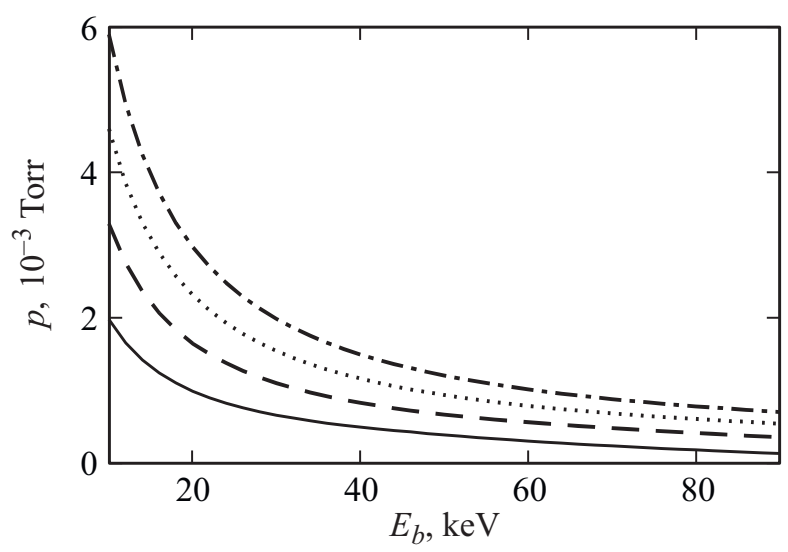

Рис. 6. Границы образования ВК в зависимости от давления нейтрального газа (Не), энергии и тока пучка. $\left|I_{b 0}\right|$ : сплошная - 300, штриховая - 500, пунктирная - 700, штрихпунктирная $-900 \mathrm{~A} ; r_{b}=2 \mathrm{~cm}, R_{c}=4.1 \mathrm{~cm}$.

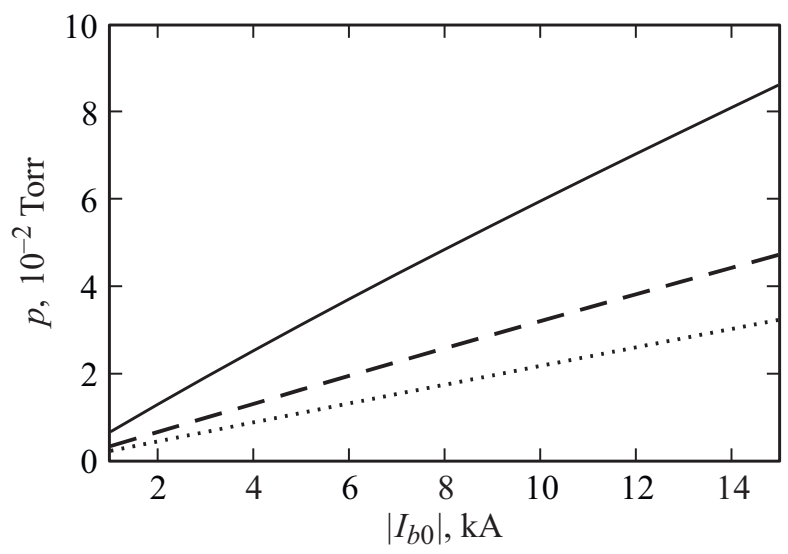

Рис. 7. Границы областей образования ВК в зависимости от давления нейтрального газа $(\mathrm{He})$, энергии и тока пучка. $\varepsilon_{b}$ : сплошная -10 , штриховая -20 , пунктирная $-30 \mathrm{keV}$; $r_{b}=2 \mathrm{~cm}, R_{c}=4.1 \mathrm{~cm}$.

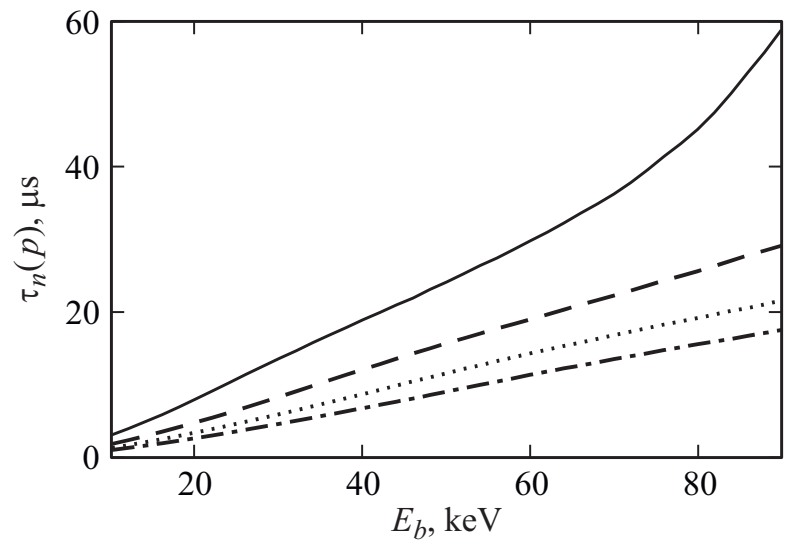

Рис. 8. Время достижения полной зарядовой нейтрализации на границе образования ВК в зависимости от давления нейтрального газа $(\mathrm{He})$, энергии и тока пучка. $\left|I_{b 0}\right|$ : сплошная 300 , штриховая - 500, пунктирная - 700, штрихпунктирная $-900 \mathrm{~A} ; r_{b}=2 \mathrm{~cm}, R_{c}=4.1 \mathrm{~cm}$.

гии электронов пучка приводят к уменьшению времени зарядовой нейтрализации на границе. А именно в случаях, когда ВК не образуется, время полной зарядовой нейтрализации тем больше, чем выше энергия электронов пучка и ниже амплитуда тока пучка. От сорта газа оно не зависит.

Численные расчеты эрозии фронта проводились также для различных геометрий трубы дрейфа. Получено, что с увеличением отношения $R_{c} / r_{b}$ потери пучка из-за эрозии увеличиваются при других постоянных параметpax, a время полной зарядовой нейтрализации на границе образования ВК уменышается. Так, при $R_{c} / r_{b}=2$, $\varepsilon_{b}=20 \mathrm{keV}, I_{b 0}=-15 \mathrm{kA}$ в аргоне требуется давление газа не ниже $10^{-2}$ Torr, чтобы избежать эрозии. А при $R_{c} / r_{b}=3$ давление должно быть свыше $1.4 \cdot 10^{-2}$ Torr. Время полной зарядовой нейтрализации на границе при этих же параметрах и увеличении $R_{c} / r_{b}$ с двух до трех уменьшится с 0.16 до $0.12 \mu \mathrm{s}$.

В аргоне граница образования ВК смещается в сторону меньших давлений по сравнению с гелием. В слабоионизованном газе для фиксированных параметров системы сдвиг границы по давлению (рис. 6, 7) соответствует понижению давления в 5 раз. Это связано с тем, что сечение ионизации аргона выше сечения ионизации гелия в $\sigma_{i b}(\mathrm{Ar}) / \sigma_{i b}(\mathrm{He}) \approx 5$ раз в диапазоне энергий в несколько десятков $\mathrm{keV}$, а вклад плазменных электронов в ионизацию газа достаточно мал. Большее сечение ионизации аргона приводит также к тому, что в гелии ВК образуется раньше и исчезает позже.

\section{Заключение}

Предложенная математическая модель адекватно описывает процессы ионизации газа и эрозии тока пучка в условиях неполной зарядовой нейтрализации. Она позволяет в широком диапазоне параметров при наличии 
ВК определять длительность эрозии переднего фронта для низкоэнергетических пучков во внешнем магнитном поле.

Показано, что в рассматриваемом диапазоне параметров ВК не будет образовываться при $p>0.1$ Torr.

В отсутствие эрозии время полной зарядовой нейтрализации обратно пропорционально энергии электронов пучка. Вклад плазменных электронов в ионизацию газа до полной зарядовой нейтрализации пучка достаточно мал.

\section{Список литературы}

[1] Серов А.А. // Физика плазмы. 2009. Т. 35. Вып. 7. С. 624 635.

[2] Пушкарев А.И., Ремнев Г.Е., Пономарев Д.В., Ежсов В.В., Гончаров Д.В. // Известия ТПУ. 2006. Т. 309. Вып. 2. C. $103-108$.

[3] Русанов В.Д., Фридман А.А. Физика химически активной плазмы. М.: Наука, 1984. 416 с.

[4] Sharp W.M., Lampe M. // Phys. Fluids. 1980. Vol. 23. N 12. P. 2383-2395.

[5] Lee E.P., Cooper R.K. // Part. Accel. 1976. Vol. 7. P. 83-95.

[6] Глазычев Л.В., Норман Г.Е., Сорокин Г.А. // Письма в ЖТФ. 1989. Т. 15. Вып. 1. С. 3-7.

[7] Глазычев Л.В., Сорокин Г.А. // Физика плазмы. 1990. Т. 16. Вып. 3. С. 370-375.

[8] Богданкевич Л.С., Рухадзе А.А. // УФН. 1971. Т. 103. Вып. 4. С. 609-640.

[9] Озур Г.Е., Батраков А.В., Карлик К.В., Зюлькова Л.А. // Изв. вузов. Физика. 2013. Т. 56. Вып. 7-2. С. 37-41.

[10] Григорьев С.В., Девятков В.Н., Миков А.В., Москвин П.В., Тересов А.Д. // Изв. вузов. Физика. 2014. Т. 57. Вып. 11-3. C. 58-62.

[11] Диденко А.Н., Григорьев В.П., Усов Ю.П. Мощные электронные пучки и их применение. М.: Атомиздат, 1977. $280 \mathrm{c}$.

[12] Gryzinski M. // Phys. Rev. 1965. Vol. 138. N 2A. P. 336-358. 